\title{
Enhanced efficacy of chemically modified curcumin in experimental periodontitis: systemic implications
}

This article was published in the following Dove Press journal: Journal of Experimental Pharmacology

\author{
Howard H Wang' \\ Hsi-Ming Lee ${ }^{2}$ \\ Veena Raja ${ }^{2}$ \\ Wei Hou ${ }^{3}$ \\ Vincent J lacono ${ }^{4}$ \\ Joseph Scaduto ${ }^{5}$ \\ Francis Johnson ${ }^{6,7}$ \\ Lorne M Golub ${ }^{2}$ \\ Ying $\mathrm{Gu}^{8}$
}

'Department of Periodontology and Endodontology, School of Dental Medicine, State University of New York at Buffalo, Buffalo, NY, USA; ${ }^{2}$ Department of Oral Biology and Pathology, School of Dental Medicine, Stony Brook University, Stony Brook, NY, USA; ${ }^{3}$ Department of Preventive Medicine, School of Medicine, Stony Brook University, Stony Brook, NY, USA; ${ }^{4}$ Department of Periodontology, School of Dental Medicine, Stony Brook University, Stony Brook, NY, USA; ${ }^{5}$ Traverse Biosciences Inc., Stony Brook, NY, USA; ${ }^{6}$ Department of Chemistry, Stony Brook University, Stony Brook, NY, USA; ${ }^{7}$ Department of Pharmacological Sciences, Stony Brook University, Stony Brook, NY, USA; ${ }^{8}$ Department of General Dentistry, School of Dental Medicine, Stony Brook University, Stony Brook, NY, USA

Correspondence: Ying Gu Department of General Dentistry, School of Dental Medicine, Stony Brook University, 124 Westchester Hall, Stony Brook, NY I I794-8706, USA

$\mathrm{Tel}+\mathrm{I} 63 \mathrm{I} 6323956$

Fax + I 63I 6329705

Email ying.gu@stonybrook.edu
Introduction: Dental microbial biofilm initiates gingival inflammation, and its suppression is the current dominant strategy for treating periodontitis. However, the host response to the biofilm is largely responsible for the connective tissue breakdown including alveolar bone loss, which is mediated by proinflammatory cytokines and matrix metalloproteinases (MMPs). Methods: The current study compared the efficacy of a novel host-modulation compound, a chemically modified curcumin (CMC 2.24), to that of its parent compound (natural curcumin), in both lipopolysaccharide (LPS) (a bacterial endotoxin)-induced cell culture and in vivo models of periodontitis.

Results: In cell culture, both CMC 2.24 and curcumin appeared similarly effective in suppressing LPS-induced cytokine (IL-1 $\beta$ and TNF- $\alpha$ ) secretion by mononuclear inflammatory cells; however, CMC 2.24 significantly reduced MMP-9 secretion by $78 \%(P<0.05)$ whereas curcumin was ineffective. In vivo, CMC 2.24 administration was more effective than curcumin in suppressing (a) IL-1 $\beta$ in gingival tissue and (b) MMP-9 in both gingiva and plasma, the latter indicating a reduced severity of systemic inflammation. The difference in primary clinical outcome between the two treatments was that CMC 2.24 reduced the pathologically excessive alveolar bone loss, assessed morphometrically at multiple sites, by $80 \%-90 \%(P<0.01)$, whereas curcumin, surprisingly, either increased $(P<0.05)$ or had no effect on alveolar bone loss at these sites.

Conclusion: These data, plus that from previous studies, support the therapeutic potential of CMC 2.24 in the management of inflammatory periodontal disease and its ability to reduce the risk of associated systemic diseases. The current study also indicates that the MMP-9 inhibitor efficacy is associated with the ability of CMC 2.24 (but not curcumin) to inhibit alveolar bone loss in this rat model of periodontitis.

Keywords: chemically modified curcumin, cytokines, matrix metalloproteinases, MMPs, periodontitis, rats

\section{Introduction}

Periodontitis, the most common of all chronic inflammatory diseases, is initiated by infection with different types of oral bacteria, notably the "red complex," which includes Porphyromonas gingivalis, Tannerella forsythia, Prevotella intermedia, and other Gram-negative anaerobes organized as a dental biofilm. ${ }^{1}$ However, the breakdown and loss of the periodontal connective tissues including the alveolar bone is largely the result of the host response to this biofilm. ${ }^{2}$ The host response mediators include proinflammatory cytokines such as IL-1 $\beta$, TNF- $\alpha$, IL-6, and the arachidonic acid metabolites such as the prostaglandins. In addition, the degradation of the collagen fibers (mostly type I and type III collagens), proteoglycan ground substance, 
and other constituents of the periodontium are mediated by neutral proteinases especially the matrix metalloproteinases (MMPs), notably leucocyte-type collagenase (MMP-8) and gelatinase (MMP-9). ${ }^{3}$

Current treatment modalities for chronic periodontitis include scaling and root planing and sometimes include the application of adjunctive therapy such as topical antimicrobials, eg, chlorhexidine, minocycline microspheres, taurolidine gels, and more recently, desiccants used for oral tissue decontamination as well as a variety of periodontal surgical techniques. ${ }^{47}$ The routine use of adjunctive systemic antibiotics is now contraindicated because of the all-too-common emergence of antibiotic-resistant bacteria and Clostridium difficile colitis, with potential for fatal consequences. ${ }^{8,9}$

Therapeutic modulation of the host response is another strategy for the treatment of chronic periodontitis. Currently, the only FDA-approved medication for this condition is a nonantibiotic formulation of doxycycline, which functions as an MMP-inhibitor. ${ }^{10}$ This formulation $(20 \mathrm{mg}$, twice a day, in contrast to the antimicrobial dose of $100 \mathrm{mg}$ qd or bid) is now generic (off-patent) and an updated nonantibiotic doxycycline version (40 mg, sustained release, one daily dose) - a novel formulation exhibiting similar pharmacokinetics - is currently widely used in dermatology. ${ }^{11}$

The efficacy of the non-antimicrobial dose of doxycycline as an adjunctive treatment for periodontitis is well proven..$^{12,13}$ Additional host-modulation medications for the management of periodontitis are needed; a promising category of compounds are novel chemically modified curcumins. The natural compound, curcumin (derived from the spice, turmeric), has been studied for decades for its wide-ranging health benefits. ${ }^{14-16}$ Recently, we modified curcumin by making it triketonic, rather than diketonic, to increase its efficacy as a zinc (and calcium)-binder and a more potent MMP-inhibitor. ${ }^{17}$ This compound, a phenylaminocarbonyl curcumin, chemically modified curcumin (CMC 2.24), has been reported to effectively reduce MMP activity in vitro, in cell culture, and in several animal models of periodontitis, cancer, diabetes, acute respiratory distress syndrome, and impaired wound healing. ${ }^{18-21}$

Although many studies have demonstrated natural curcumin's ability to reduce inflammatory cells and cytokines in diseases such as periodontitis, they have not observed a significant inhibition of bone loss. ${ }^{22,23}$ Therefore, the aim of this study was to compare the efficacy of the novel compound, CMC 2.24 with that of natural curcumin in the treatment of experimental periodontitis in rats with the null hypothesis that there is no difference in efficacy between the two drugs in terms of inhibition of bone loss. The primary outcome measurement of drug efficacy was to assess both radiographic and morphometric alveolar bone loss, with secondary outcome measurements of levels of proinflammatory cytokines and MMP activity in cultured inflammatory cells, gingiva, plasma, and serum.

\section{Materials and methods \\ Chemical reagents}

All chemical reagents, lipopolysaccharide (LPS) from P. gingivalis, and curcumin were purchased from Sigma-Aldrich Co. (St. Louis, MO). All cell culture reagents were purchased from Thermo Fisher Scientific (Waltham, MA, USA) CMC 2.24 was provided by Chem-Master Intl. Inc. (99.5\% pure, Hauppauge, NY, USA); see Figure 1.

\section{Cell culture assay}

Human peripheral blood mononuclear cells (PBMCs) were isolated and cultured as described previously. ${ }^{21}$ The human PBMCs were isolated from the commercially available human leukocyte pack with no donor information and is exempted from the institutional review board. Either LPS derived from $P$. gingivalis $(50 \mathrm{ng} / \mathrm{mL})$ or vehicle alone were added to the culture for 18 hours. Curcumin or CMC 2.24 was added at a final concentration of 2 or $5 \mu \mathrm{M}$. The conditioned media from the cultures were then analyzed for the presence of proinflammatory cytokines, TNF- $\alpha$, IL-1 $\beta$, IL-6, MCP-1, and $\mathrm{PGE}_{2}$ using ELISA, and for MMP-9 using ELISA and gelatin zymography (both MMP-2 and MMP-9, see below) as described by us previously. ${ }^{17,19}$

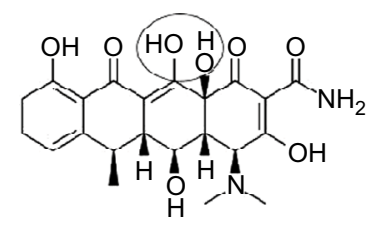

Doxycycline (diketonic)
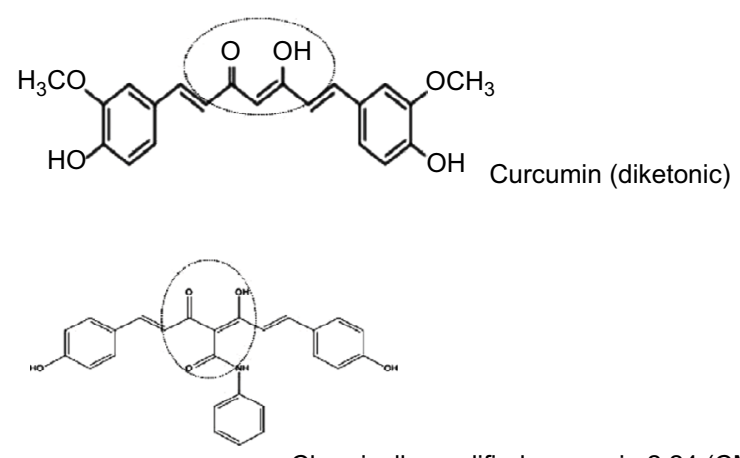

Chemically modified curcumin 2.24 (CMC 2.24) (triketonic)

Figure I The molecular structures of doxycycline (top), curcumin (middle), and CMC 2.24 [I,7-bis-(4-hydroxyphenyl)-4-phenylaminocarbonyl-IE,6E-heptadien-3,5dione] (bottom).

Abbreviation: CMC, chemically modified curcumin. 


\section{Animal studies}

Protocols for animal studies were approved by Stony Brook University's Institutional Animal Care and Use Committee (IACUC \#230617). Animals were housed in the Division of Laboratory Animal Resources at Stony Brook University, with care provided by the center's personnel. All procedures were conducted at the same location. This facility follows the Animal Welfare Act (USDA enforced), the Public Health Service Act (OLAW enforced), and NY State law (DOH enforced), and is an AAALAC International accredited facility.

Twenty male Sprague Dawley rats (275-300 g body weight; viral antibody free; Charles River Labs, Wilmington, MA) were distributed into the control and experimental groups ( $\mathrm{n}=5$ rats per group). Then $30 \mu \mathrm{g}$ of lipopolysaccharide (LPS) from E. coli (Strain 055:B5; Sigma Chem Co., St. Louis, MO, USA) diluted in $3 \mu \mathrm{L}$ of PBS was injected into the palatal gingiva between the upper first and second molars using a Hamilton microsyringe (Agilent, Santa Clara, CA, USA) to induce experimental periodontitis as described previously. ${ }^{24}$ Injections with LPS endotoxin for 2 weeks have been shown in previous studies by us and other researchers to induce experimental periodontitis in rats. ${ }^{19,24,25}$ Control group rats received PBS injection at the same site as the experimental groups. PBS and LPS injections were given three times a week for 2 weeks. Group 1 rats received placebo injections with PBS and were orally gavaged with vehicle alone. Groups 2,3 , and 4 received LPS injections; however, group 2 rats were orally gavaged with the vehicle alone, whereas group 3 rats received curcumin $30 \mathrm{mg} / \mathrm{kg}$, and group 4 rats received 30 $\mathrm{mg} / \mathrm{kg} \mathrm{CMC} \mathrm{2.24.} \mathrm{PBS} \mathrm{and} \mathrm{LPS} \mathrm{injections} \mathrm{were} \mathrm{administered}$ at the same time as the daily oral administration of vehicle alone, curcumin, or CMC 2.24 (Figure 2).

Experiment 1: "Prophylatic" model

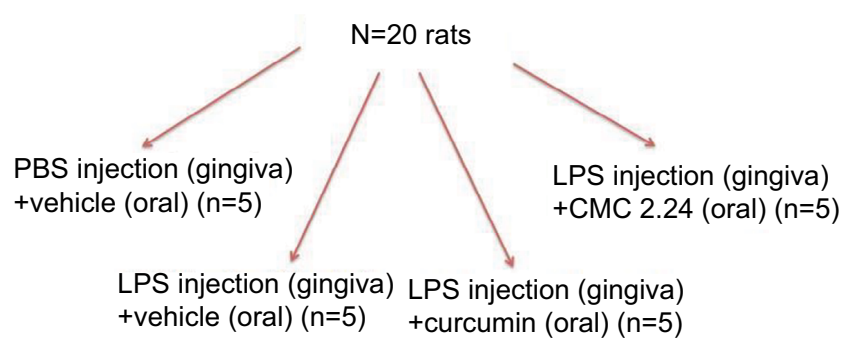

Rats were injected with PBS or LPS for 2 weeks and orally gavaged for 2 weeks at the same time as experimental periodontitis was initiated and perpetuated.

Figure 2 Outline of experimental groups and timeline. Abbreviation: LPS, lipopolysaccharide.
At the end of the experimental period, the animals were sacrificed and gingival tissue and maxillary jaw bones were collected. The gingival tissues were excised around maxillary first and second molars of each rat and pooled per experimental group (five rats per group) as insufficient gingival tissue were obtained from each rat for individual analysis. The gingival tissues were used for extraction and partial purification of MMPs as described by us previously. ${ }^{26,27}$ Briefly, the gingival samples were homogenized, and supernatants were collected and dialyzed exhaustively against $50 \mathrm{mM}$ Tris buffer ( $\mathrm{pH} 7.8$ ) containing $0.2 \mathrm{M} \mathrm{NaCl}$ and $5 \mathrm{mM} \mathrm{CaCl}_{2}$. Ammonium sulfate was added to the dialysate to produce $60 \%$ saturation, allowed to stand overnight, and the precipitate containing the MMPs was collected by centrifugation at $15,000 \mathrm{rpm}$ for 90 minutes. The pellets were then dissolved in Tris buffer ( $\mathrm{pH}$ 7.8) containing $\mathrm{NaCl}$, $\mathrm{CaCl}_{2}$, and $0.05 \%$ Brij solution and exhaustively dialyzed against the same buffer.

In addition, blood samples were collected and the serum and plasma were separated by standard procedure and analyzed for MMPs and cytokines as described below.

\section{Gelatin zymography}

Gelatin zymography was carried out as described by us previously. ${ }^{21}$ Briefly, polyacrylamide was copolymerized with gelatin at a final concentration of $1 \mathrm{mg} / \mathrm{mL}$. After loading, the samples were electrophoresed at $120 \mathrm{~V}$, followed by staining with Coomassie Brilliant Blue R-250. MMP-2 and MMP-9 standards were purchased from R\&D Systems, Inc. (Minneapolis, MN). Clear zones of lysis against a blue background indicated gelatinolytic activity and were analyzed by an image processing program, ImageJ, to assess gelatinase activity. ${ }^{21}$ Analysis of the gelatinolytic bands was carried out by a single-blinded investigator as previously described by Hu et al. (2010). ${ }^{28}$

\section{ELISA assay}

ELISA kits for TNF- $\alpha$, IL- $1 \beta$, and IL-6, were purchased from R\&D Systems, Inc. The assays were carried out according to the manufacturer's instructions and followed a previously published protocol. ${ }^{29}$ The results were subsequently normalized to the concentration of the protein in the samples using the Bio-Rad protein assay (Hercules, CA).

\section{Morphometric analysis of alveolar bone loss}

The soft tissues were carefully dissected to maintain the integrity of the maxillary bone specimens. ${ }^{30}$ The jaws were 
then completely defleshed by immersion in boiling water for 20 minutes, then in $2 \mathrm{~N}$ sodium hydroxide for 10-15 minutes, followed by gentle mechanical dissection of the remaining soft tissue. After washing in running water, the specimens were dried overnight. A single independent examiner, blinded to the experimental groups, carried out all morphometric measurements of alveolar bone loss on each surface by measuring the distance between the cemento-enamel junction (CEJ) to alveolar bone crest at four positions, namely positions $6,7,8$, and 9 on the first and second molars, using ImageJ analysis software (Figure 3) after the pictures were acquired using a DSLR camera at a fixed angle. A millimeter ruler was placed adjacent to the sample during the photograph acquisition for calibration in ImageJ software. Two other independent examiners validated the reproducibility of the results after a blind study was conducted and the inter-rater reliability score (kappa score) was calculated to be 0.88 .

\section{Statistical analysis}

We have reported a difference of $0.1 \mathrm{~mm}$ in the group means of the bone loss between untreated and treated rats. Assuming a similar difference in the present experiments between untreated and CMC 2.24-treated rats, a sample size of five per group will allow us to determine if significant differences are present between groups assuming a two-sided $\alpha$ level of 0.05 and a power $>99 \%$.

Cytokine and MMP level differences in cell culture between groups were analyzed by Student's $t$-test, with $P \leq 0.05$ taken as statistically significant, using Microsoft Excel (Redmond, WA). In the rat animal studies, when comparing two groups (normal vs LPS; LPS vs CMC 2.24), Student's $t$-test was used as well; $P$-values $\leq 0.05$ were considered statistically significant.

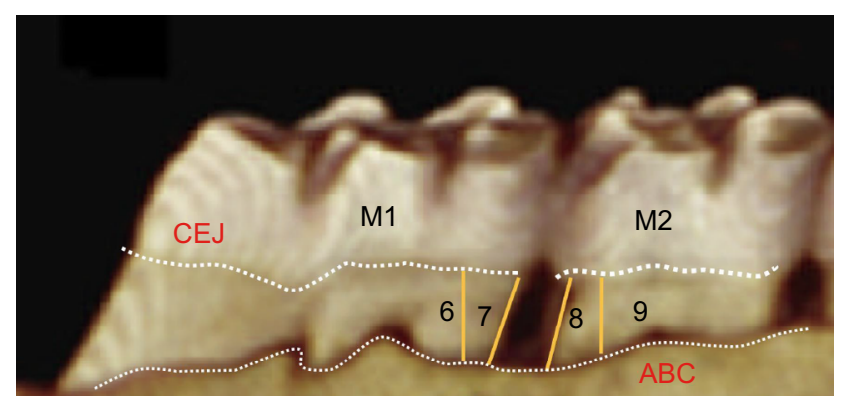

Figure 3 Diagram indicating the positions of the key study region of maxillary molar teeth in rats at positions $6,7,8$, and 9 .

Abbreviations: $A B C$, alveolar bone crest; $C E$ J, cemento-enamel junction; $M I$, first molar; M2, second molar.

\section{Results \\ Cell culture study}

As shown in Figure 4A, B, in the cell culture studies, human monocytes were incubated in serum-free conditioned media (SFCM) $\left(37^{\circ} \mathrm{C}, 95 \%\right.$ air, $\left.5 \% \mathrm{CO}_{2}\right)$ for 18 hours with or without LPS (P. gingivalis, $50 \mathrm{ng} / \mathrm{mL}$ ). Curcumin and CMC 2.24 were added to selected wells at concentrations of 2 and $5 \mu \mathrm{M}$. In the absence of CMC 2.24 and LPS, monocytes secreted about $200 \mathrm{pg} / \mathrm{mL}$ of TNF- $\alpha$, which increased to $1470 \mathrm{pg} /$ $\mathrm{mL}$ when LPS was added to the culture. TNF- $\alpha$ levels were reduced by $11 \%$ and $39.6 \%$ with 2 and $5 \mu \mathrm{M}$ curcumin addition, respectively; however, only the latter value was statistically significant $(P<0.05)$. In contrast, when $\mathrm{CMC}$ 2.24 was added to the cultures of the LPS-stimulated monocytes, in final concentrations of 2 and $5 \mu \mathrm{M}$, a greater effect was seen; the extracellular TNF- $\alpha$ levels were decreased by $48 \%(P<0.05)$ and $39 \%(P<0.05)$, respectively. In addition, when monocytes were challenged with LPS, they secreted $185 \mathrm{pg} / \mathrm{mL}$ of IL-1 $\beta$ while control cells secreted only about $12 \mathrm{pg} / \mathrm{mL}$ (Figure 5A, B). IL-1 $\beta$ levels were reduced by $53 \%$ and $78 \%$ with 2 and $5 \mu \mathrm{M}$ curcumin, respectively $(P<0.05)$. When these cells were incubated with CMC $2.24(2 \mu \mathrm{M})$ IL- $1 \beta$ levels were decreased by $37 \%(P<0.05)$ (Figure 5$)$. In addition, the extracellular levels of MMP-9 in the SFCM from these monocyte cultures, in the presence or absence of 2 and $5 \mu \mathrm{M}$ CMC 2.24 , were analyzed by gelatin zymography and quantified by ImageJ software. Similar to the results observed for TNF- $\alpha$, the extracellular levels of MMP-9 were reduced by more than $70 \%$ (Figure 6$)$ by $5 \mu \mathrm{M}$ CMC $2.24(P<0.05)$; curcumin at $5 \mu \mathrm{M}$ concentration did not have a significant effect on MMP-9 levels (Figure 6). Although CMC 2.24 was no more effective than curcumin in reducing inflammatory mediators TNF- $\alpha$ and IL- $1 \beta$ in cell culture, CMC 2.24 was effective (and curcumin was NOT) in reducing MMP-9 levels. Of importance, this pattern was also seen in vivo systemically in the circulation (see later).

\section{In vivo studies}

\section{Gingival tissue}

After the 2-week protocol, the LPS-injected rats were orally administered either curcumin or CMC 2.24 (30 mg/kg). Gingival tissue was obtained and pooled by group, as described above, since insufficient gingival tissue was available for individual analysis. Rat gingiva were pooled by group, extracted, and partially purified with urea. Samples were analyzed for MMP-2 and MMP-9 by gelatin zymography (Figure 7A) and quantitative measurements of MMP-9 and MMP-2 attained by using ImageJ software (Figure 7B, C). Total MMP-2 was 
A

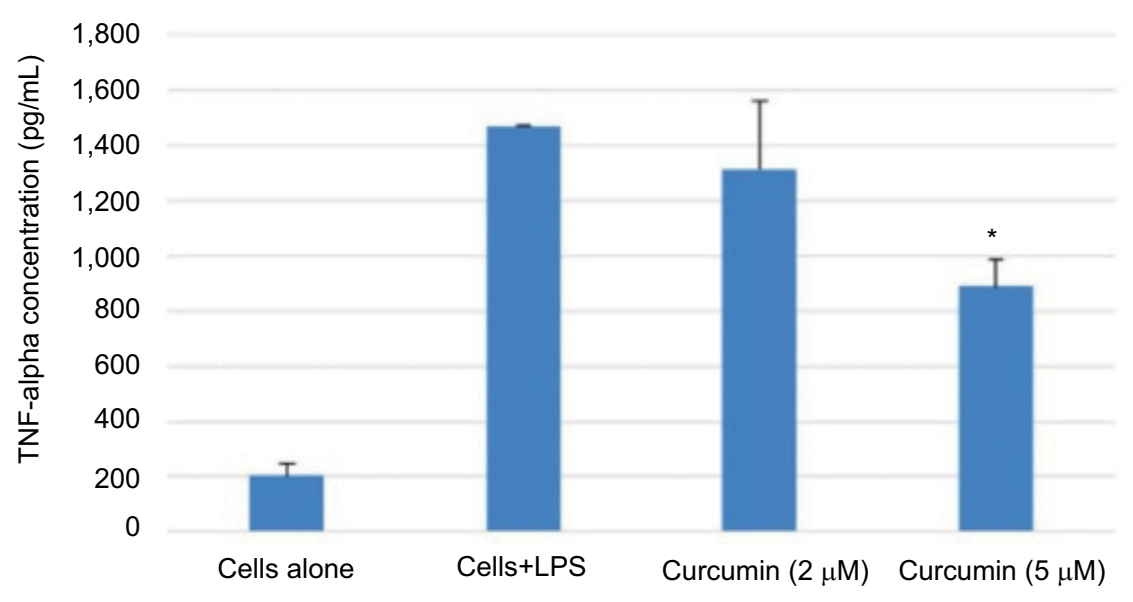

B

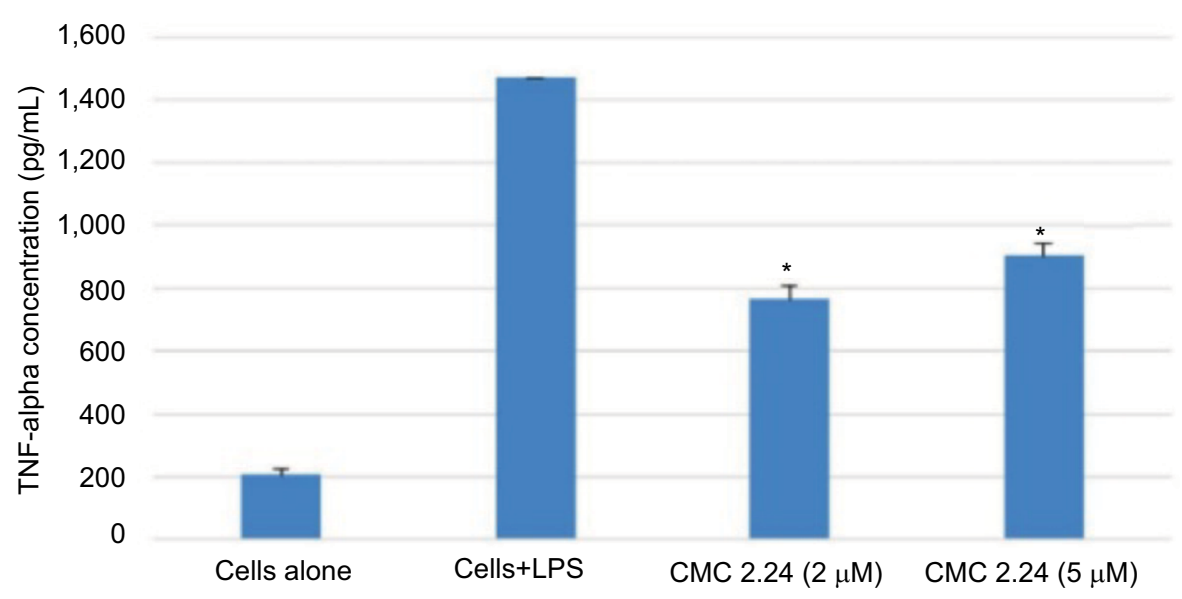

Figure 4 (A) Effect of curcumin on levels of TNF- $\alpha$ secreted by human monocytes. Human peripheral blood-derived monocytes (I $\times 10^{6}$ cells/well) were cultured in serumfree media $\left(37^{\circ} \mathrm{C}, 5 \% \mathrm{CO}_{2} / 95 \% \mathrm{O}_{2}, 18\right.$ hours $)$ with LPS $(50 \mathrm{ng} / \mathrm{mL})$ and with or without curcumin $(2$ or $5 \mu$ M). Conditioned medium was analyzed for TNF- $\alpha$ using ELISA. (B) Effect of CMC 2.24 on levels of TNF- $\alpha$ secreted by human monocytes. Human peripheral blood-derived monocytes (I $\times 10^{6}$ cells/well) were cultured in serum-free media $\left(37^{\circ} \mathrm{C}, 5 \% \mathrm{CO}_{2} / 95 \% \mathrm{O}_{2}, 18\right.$ hours $)$ with LPS $(50 \mathrm{ng} / \mathrm{mL})$ and with or without CMC 2.24 (2 or $\left.5 \mu \mathrm{M}\right)$. Conditioned medium was analyzed for TNF- $\alpha$ using ELISA. The $*$ denotes that the difference is statistically significant with $P<0.05$.

Abbreviations: CMC, chemically modified curcumin; LPS, lipopolysaccharide.

dramatically elevated in two of the three LPS groups (Figure 7A). MMP-2 appeared slightly reduced by curcumin and CMC 2.24 (Figure 7B). MMP-9 was increased by 145\% in the LPS/vehicle group (Figure 7C) when compared to that in the control group. Curcumin reduced the total MMP-9 by $31.6 \%$, while treatment with CMC 2.24 reduced the total MMP-9 back to $75 \%$ of normal when compared with the LPS/vehicle group (Figure 7C).

Cytokine levels in the gingiva were analyzed using ELISA techniques. The results show that compared to the normal group, there was a pronounced elevation of IL- $1 \beta$ levels in the LPS/vehicle group by $40 \%$, whereas in the LPS/ curcumin and LPS/CMC 2.24 groups the elevated IL-1 $\beta$ was decreased by $17.4 \%$ and $29.5 \%$, respectively, in comparison to that in the LPS/vehicle group (Figure 8). Treatment with curcumin showed modest reduction of IL-6 cytokine levels when compared with the LPS/vehicle group and the CMC 2.24-treated group was slightly more effective. No differences in the pattern of response were seen between the groups for TNF- $\alpha$ (data not shown).

\section{Plasma}

Plasma samples were prepared and analyzed for MMP-2 and MMP-9 by gelatin zymography as described by us previously. ${ }^{19}$ The differences between the groups were even more pronounced in the activated-MMP-9 analysis; the LPS group showed a $133.6 \%$ increase $(P<0.05)$ in activated MMP-9 compared to the normal group. Curcumin did not significantly reduce activated MMP-9 whereas CMC 2.24 significantly $(P<0.05)$ decreased activated MMP-9 by $84.9 \%$, 
A

250

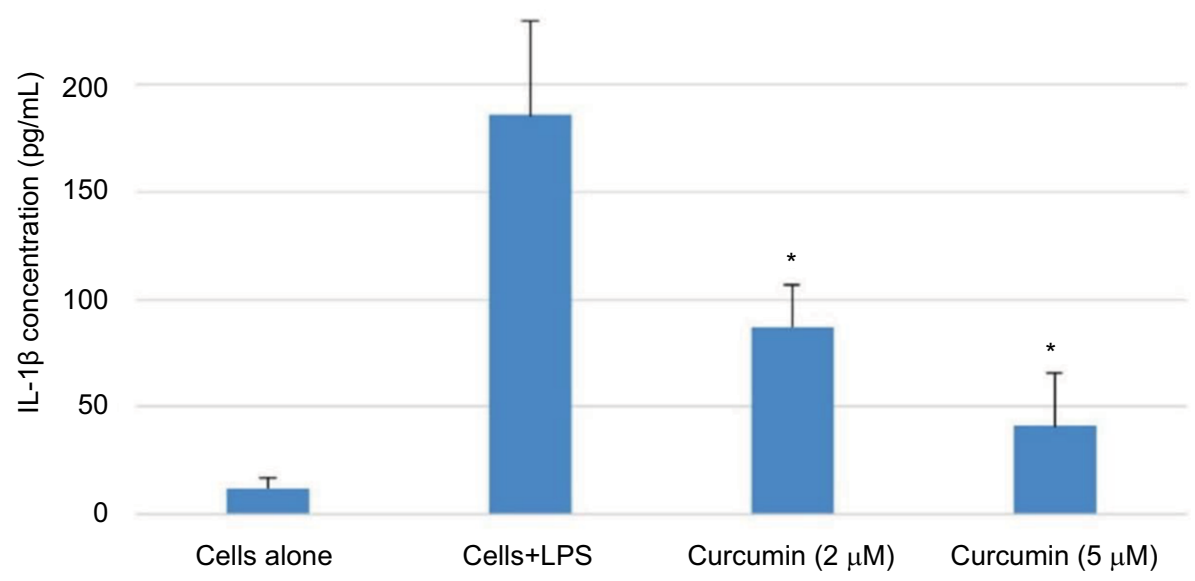

B

250

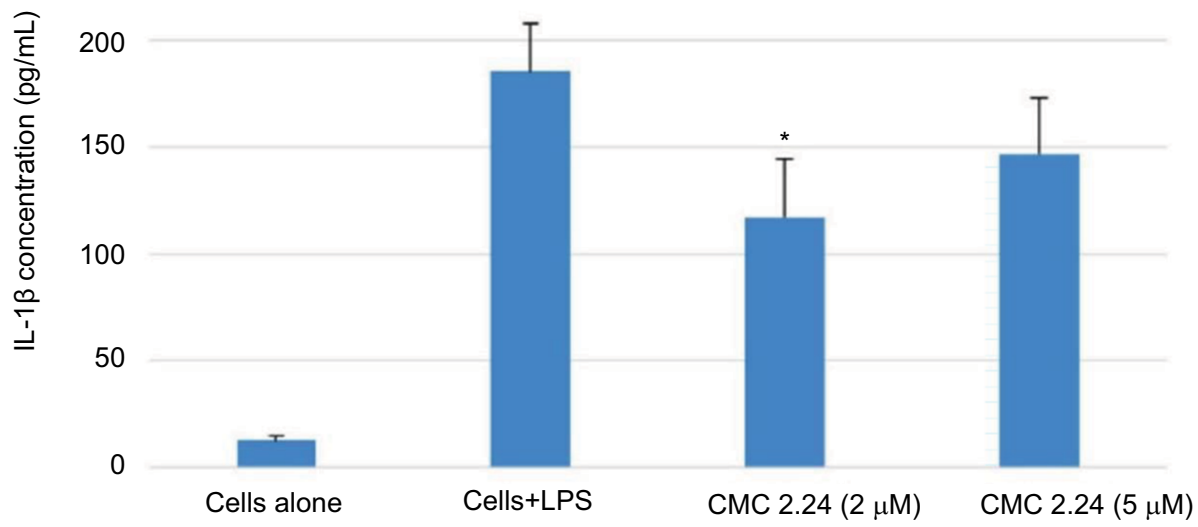

Figure 5 (A) Effect of curcumin on levels of IL-I $\beta$ secreted by human monocytes. Human peripheral blood-derived monocytes $\left(I \times 10^{6}\right.$ cells/well) were cultured in serumfree media $\left(37^{\circ} \mathrm{C}, 5 \% \mathrm{CO}_{2} / 95 \% \mathrm{O}_{2}\right.$, 18 hours) with LPS $(50 \mathrm{ng} / \mathrm{mL})$ and with or without curcumin $(2$ or $5 \mu \mathrm{M})$. Conditioned medium was analyzed for IL-I $\beta$ using ELISA. (B) Effect of CMC 2.24 on levels of IL-I $\beta$ secreted by human monocytes. Human peripheral blood-derived monocytes $\left(I \times 10^{6} \mathrm{cells} /\right.$ well) were cultured in serum-free media $\left(37^{\circ} \mathrm{C}\right.$, $5 \% \mathrm{CO}_{2} / 95 \% \mathrm{O}_{2}$, 18 hours) with LPS (50 ng/mL) and with or without CMC $2.24(2$ or $5 \mu \mathrm{M})$. Conditioned medium was analyzed for IL-I $\beta$ using ELISA. The * denotes that the difference is statistically significant with $P<0.05$.

Abbreviations: CMC, chemically modified curcumin; LPS, lipopolysaccharide.

MMP-9 analysis

2,500

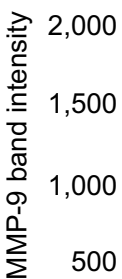

0

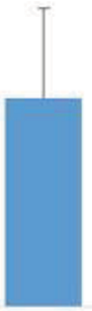

Normal

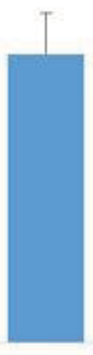

LPS

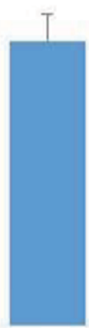

Curcumin $(2 \mu \mathrm{m}) \quad$ Curcumin $(5 \mu \mathrm{m})$
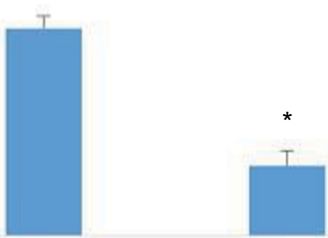

CMC $2.24(2 \mu \mathrm{m}) \quad$ CMC $2.24(5 \mu \mathrm{m})$

Figure 6 Effect of curcumin or CMC 2.24 on levels of MMP-9 secreted by human monocytes.

Notes: Human monocytes $\left(1 \times 10^{6}\right.$ cells/well) were cultured in serum-free media $\left(37^{\circ} \mathrm{C}, 5 \% \mathrm{CO}_{2} / 95 \% \mathrm{O}_{2}\right.$, 18 hours $)$ with $\mathrm{LPS}(50 \mathrm{ng} / \mathrm{mL})$ and with or without curcumin or CMC 2.24. Conditioned medium was analyzed for MMP-9 by gelatin zymography. MMP-9 levels were quantitated by Imagej. The * denotes that the difference is statistically significant with $\mathrm{P}<0.05$.

Abbreviations: CMC, chemically modified curcumin; LPS, lipopolysaccharide, MMP, matrix metalloproteinases. 
A

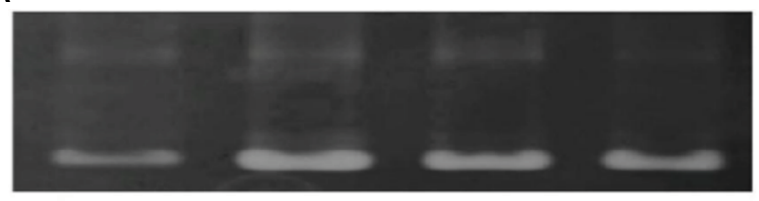

Normal LPS Curcumin $\quad$ CMC 2.24

B

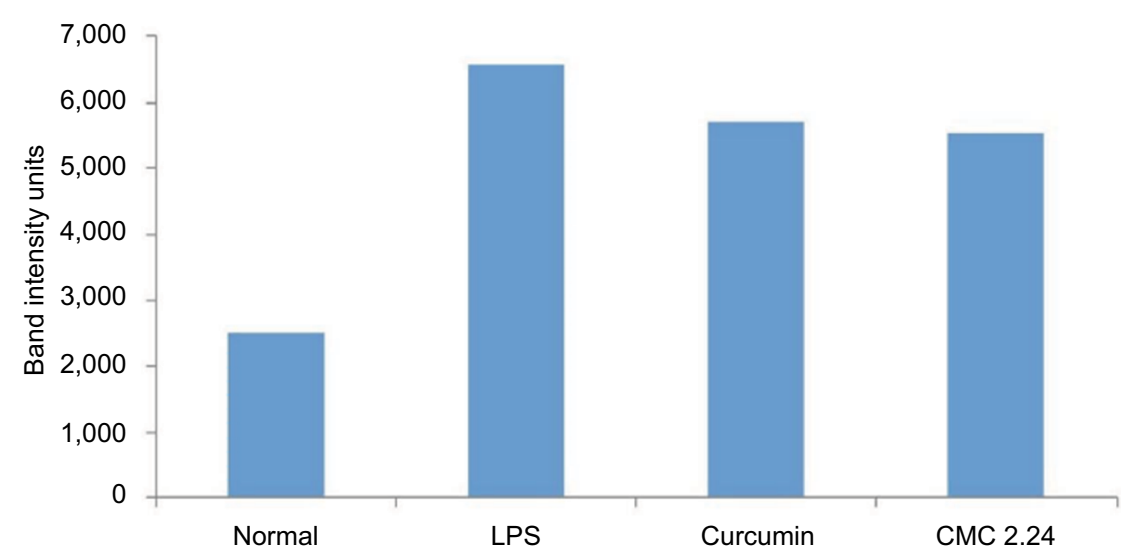

C

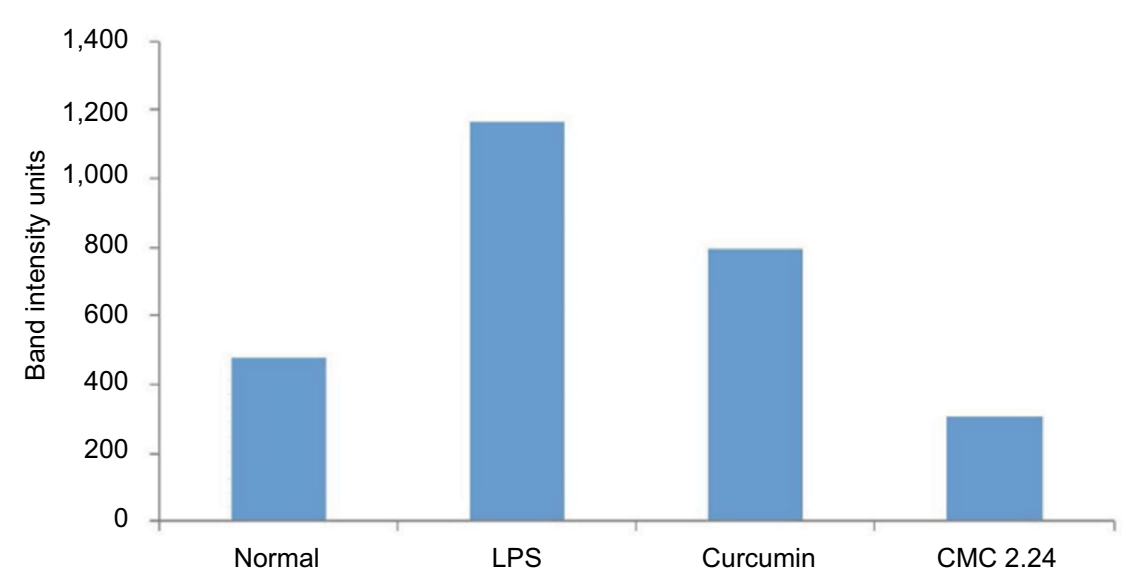

Figure 7 (A) Gel zymogram of MMP-2 and MMP-9 levels in rat gingiva. (B) Quantification of MMP-2 in rat gingiva from gel zymogram. (C) Quantification of MMP-9 in rat gingiva from gel zymogram.

Abbreviations: CMC, chemically modified curcumin; LPS, lipopolysaccharide, MMP, matrix metalloproteinases.

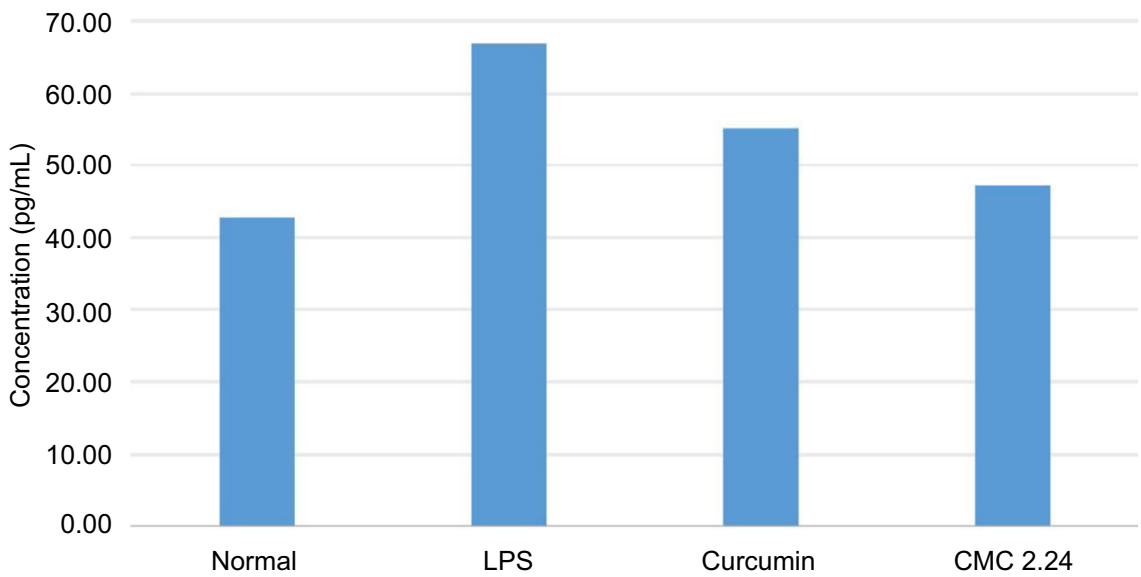

Figure 8 IL-I $\beta$ concentration in rat gingiva.

Note: Rat gingiva in each group were pooled and extracted with urea and analyzed for IL-I $\beta$ using ELISA.

Abbreviations: CMC, chemically modified curcumin; LPS, lipopolysaccharide. 
returning the level of this circulating MMP back to normal level (Figure 9). When evaluating MMP-2 levels in plasma, LPS increased its level 13\% higher than that of the normal group, but the difference was not significant $(P>0.05)$. In contrast, the LPS/curcumin group showed a $20.9 \%$ decreased level of MMP-2 compared to the LPS/vehicle group, and the LPS/CMC2.24 group reduced MMP-2 level by 54.3\%, which was statistically significant (Figure 10).

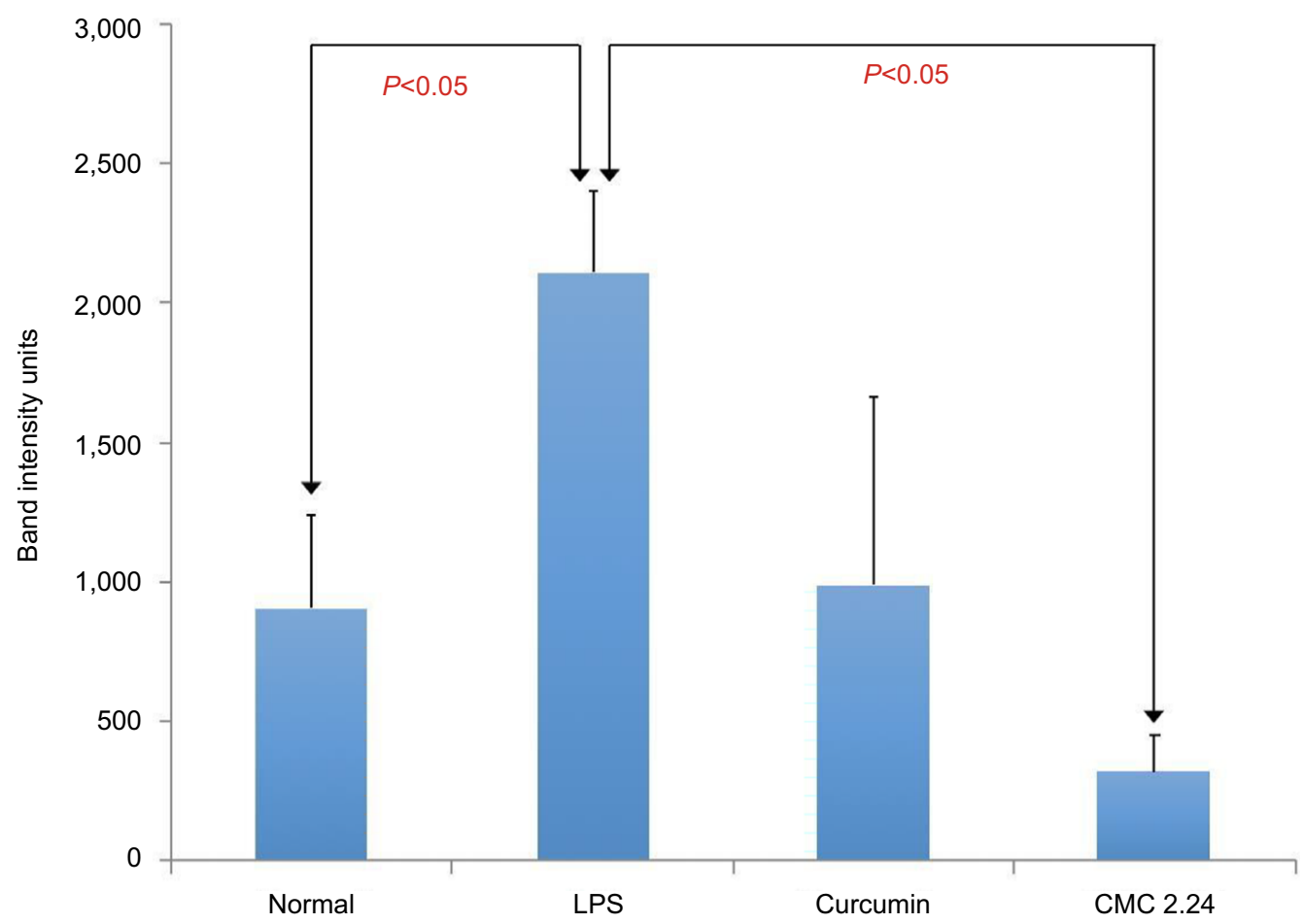

Figure 9 Activated MMP-9 levels in rat plasma.

Notes: Rat plasma were collected and analyzed through gelatin zymography. Levels of MMP-9 were quantified by ImageJ.

Abbreviations: CMC, chemically modified curcumin; LPS, lipopolysaccharide, MMP, matrix metalloproteinases.

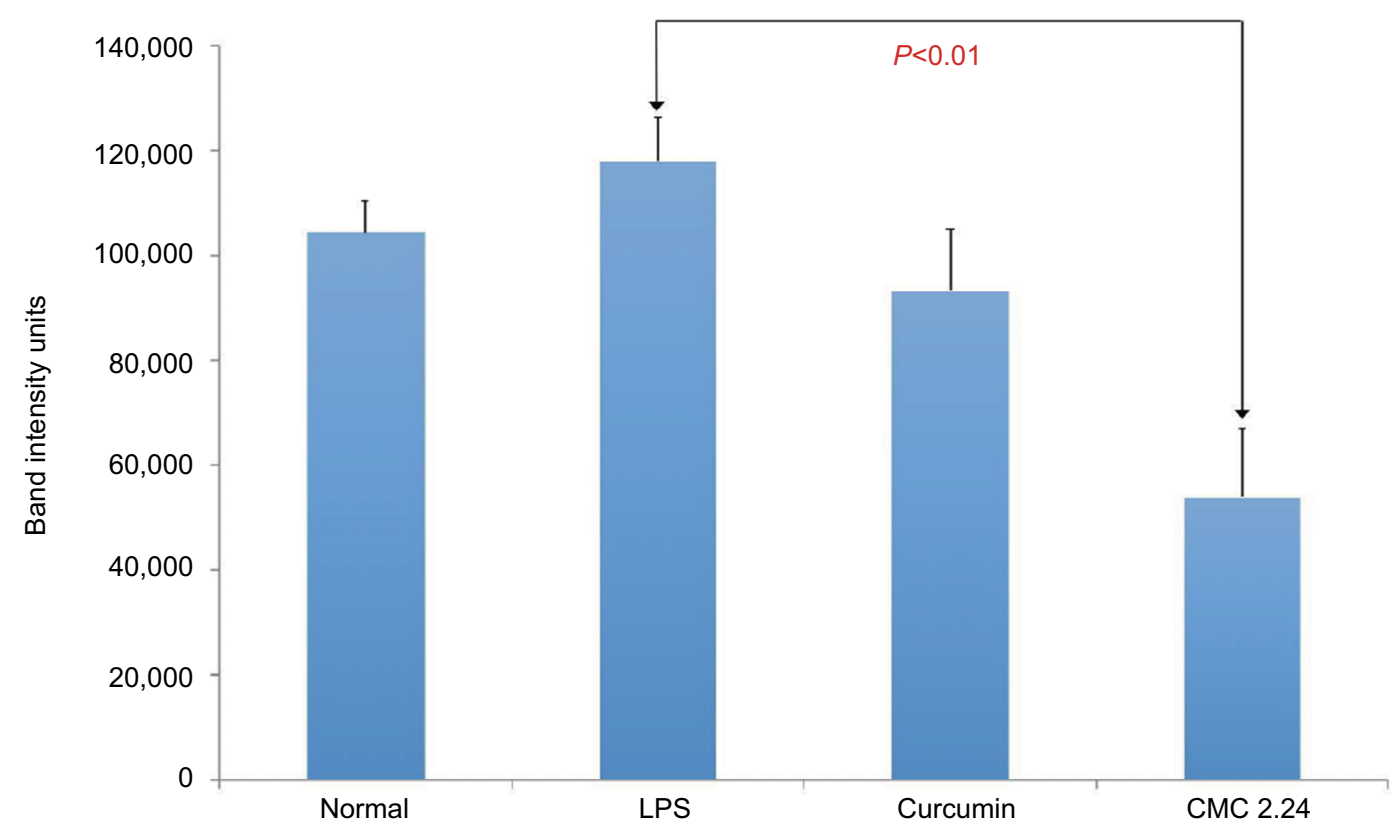

Figure 10 MMP-2 levels in rat plasma.

Notes: Rat plasma were collected and analyzed through gelatin zymography. Levels of MMP-2 were quantified by ImageJ.

Abbreviations: CMC, chemically modified curcumin; LPS, lipopolysaccharide, MMP, matrix metalloproteinases. 
In general, the LPS/vehicle group showed greater MMP levels compared to the normal group, the curcumin group, and the CMC 2.24 group, and the CMC 2.24 group appeared to exhibit the greatest reduction in MMP levels. Serum IL-1 $\beta$ levels showed a similar pattern, but the changes were not significant (data not shown).

\section{Bone morphometric and radiographic analysis}

Alveolar bone loss was measured at the maxillary first and second molars, including the interproximal regions, labeled as sites $6,7,8$, and 9 (see Figure 3 ) at both the buccal and palatal aspects of samples in each group (Figure 11) based on earlier studies by Chang et al. ${ }^{30}$ The LPS/vehicle group showed significant bone loss compared to the normal group $(P<0.01)$, and the LPS/CMC 2.24 group had the least amount of bone loss (almost back to normal levels) compared to the normal group. There was a significant reduction of $86 \%$ in pathologic bone loss measurement in the LPS/CMC 2.24 group when compared to the LPS/vehicle group $(P<0.01)$. However, interestingly, the LPS/curcumin group had the greatest amount of alveolar bone loss and this was statistically significant compared to the LPS/vehicle group $(P<0.05)$ (Figure 12). When measuring the alveolar bone loss solely on the palatal side where the PBS or LPS injections were given, similar profound effects were observed (Figure 13).

Radiographic analysis was performed on all the samples and interproximal bone loss was measured at positions 7 and 8 between the first and second maxillary molars by an

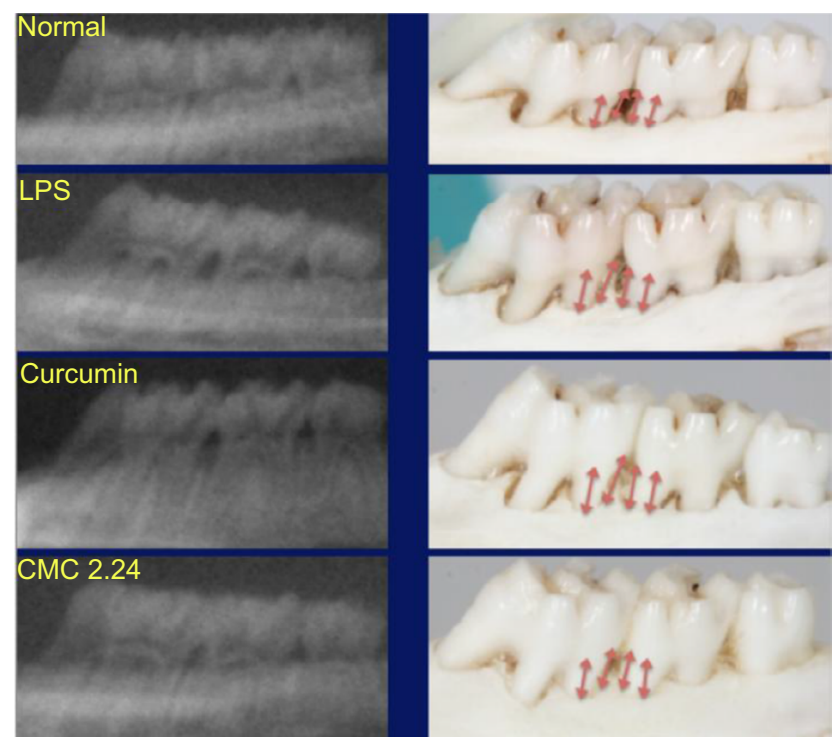

Figure I I Representative periapical radiographs and morphometric images of each group.

Abbreviations: CMC, chemically modified curcumin; LPS, lipopolysaccharide. independent examiner who was blinded to the results of the morphometric analysis. Positions 6 and 9 could not be calculated radiographically due to overlap of the teeth and the bone, meaning fewer analysis samples were included in the statistics (Figure 13). The analysis shows the same pattern of changes as the morphometric measurements. The LPS/ vehicle group had significantly greater radiographic alveolar bone height loss compared to the normal group $(P<0.01)$, while the curcumin-treated group had even greater bone loss than the LPS/vehicle group of $52 \%(P<0.05)$. The CMC 2.24-treated group showed reduction of radiographic alveolar bone height loss of $67 \%$ compared to the LPS/vehicle group but this difference was not statistically significant $(P>0.05)$ (Figure 14). However, the CMC 2.24 group exhibited bone loss that was not significantly different from that of the "normal" group.

\section{Discussion}

Host modulation is considered an important component of the three-prong approach to comprehensive periodontal therapy alongside antimicrobial control and risk/behavior modification. ${ }^{5}$ A variety of medications capable of host modulatory effects have been studied in the past including nonsteroidal anti-inflammatory drugs (ketoprofen, flurbiprofen), bisphosphonates, and different classes of tetracycline and chemically modified tetracyclines. The systemic application of nonsteroidal anti-inflammatory drugs, bisphosphonates, and antibiotics lead to significant health issues and were eventually ruled out as inappropriate host modulatory medications for the treatment of periodontitis except for non-antimicrobial-dose doxycycline, a member of the tetracycline family. ${ }^{10}$ Molecular analysis showed that host modulation effects of tetracycline were the result of the calcium- and zinc-binding $\beta$-diketone moiety at carbon 11 and $12 .{ }^{10}$ Many molecules containing similar active sites, such as thermorubin, bis-aroyl methanes, and curcumins, were investigated; however, thermorubin and bis-aroyl methanes were not suitable candidates due to their antimicrobial activity and inept modulatory capabilities. ${ }^{31,32}$ Based on preliminary results, curcumin was selected for further investigation. ${ }^{17}$ Curcumin, although is unrelated to tetracyclines, contains the same 1,3-diketonic moiety and has been known for its anti-inflammatory properties for a long time. A strategy to chemically modify curcumin to enhance its zinc-binding active sites as well as increase its stability in serum albumin is currently being developed in our lab. ${ }^{29}$ Preliminary cell culture studies showed great promise as certain variations of chemically modified curcumin were even more potent than doxycycline in inhibiting proinflammatory 


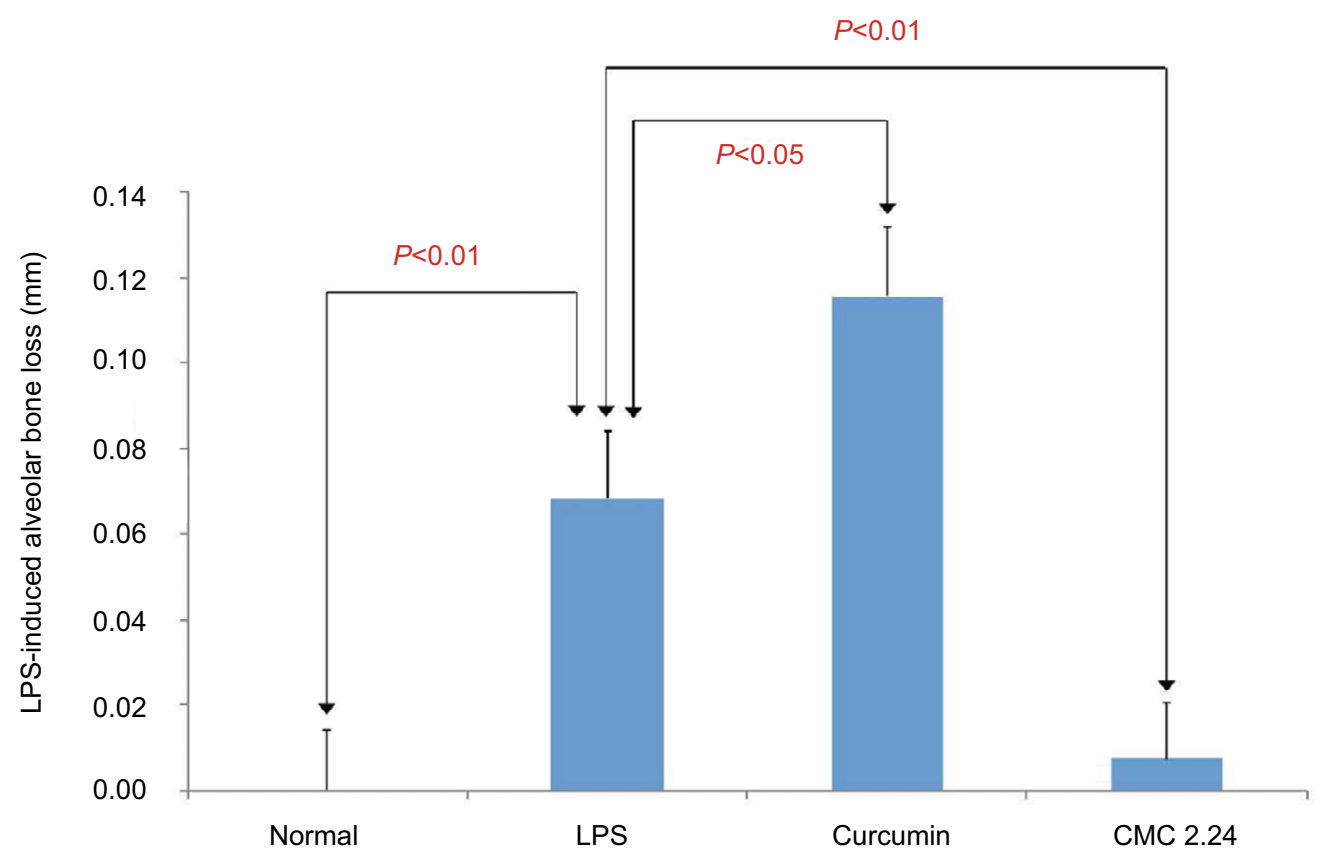

Figure 12 Morphometric analysis of maxillary bone loss at positions 6, 7, 8, and 9 on both the buccal and palatal aspects between the first and second molars. Note: The maxillae were defleshed, and bone loss was measured between first and second molars at positions 6, 7, 8, and 9 .

Abbreviations: CMC, chemically modified curcumin; LPS, lipopolysaccharide.

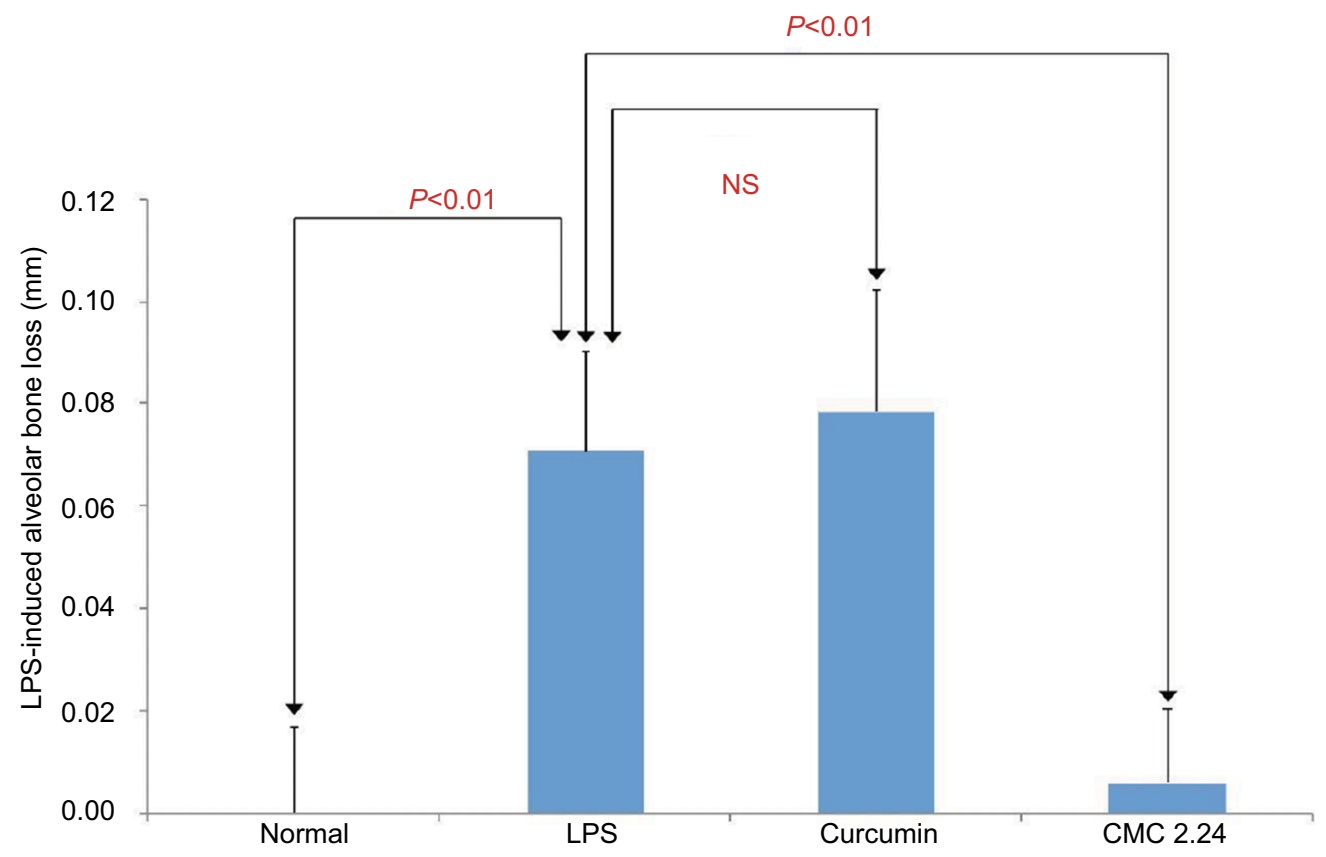

Figure 13 Morphometric analysis of maxillary bone loss at positions 6, 7, 8, and 9only on the palatal aspect between the first and second molars. Note: The maxillae were defleshed, and bone loss was measured between first and second molars at positions 6, 7, 8, and 9 .

Abbreviations: CMC, chemically modified curcumin; LPS, lipopolysaccharide; NS, not significant.

cytokines, indicating that they can be a potential host immune modulator for the treatment of chronic periodontitis.

Out of the many synthesized derivatives of curcumin, CMC 2.24 was chosen as the lead compound due to its superior capabilities in vitro, in cell culture, and in vivo in the rat model. ${ }^{17}$ In an in-vitro study, it was found that not only does $\mathrm{Zn}^{2+}$ bind more strongly to CMC 2.24 compared to curcumin, but CMC 2.24 also exhibited increased acidity and stronger binding to bovine serum albumin than curcumin, which increases its biological activity. ${ }^{29}$ In addition, CMC 


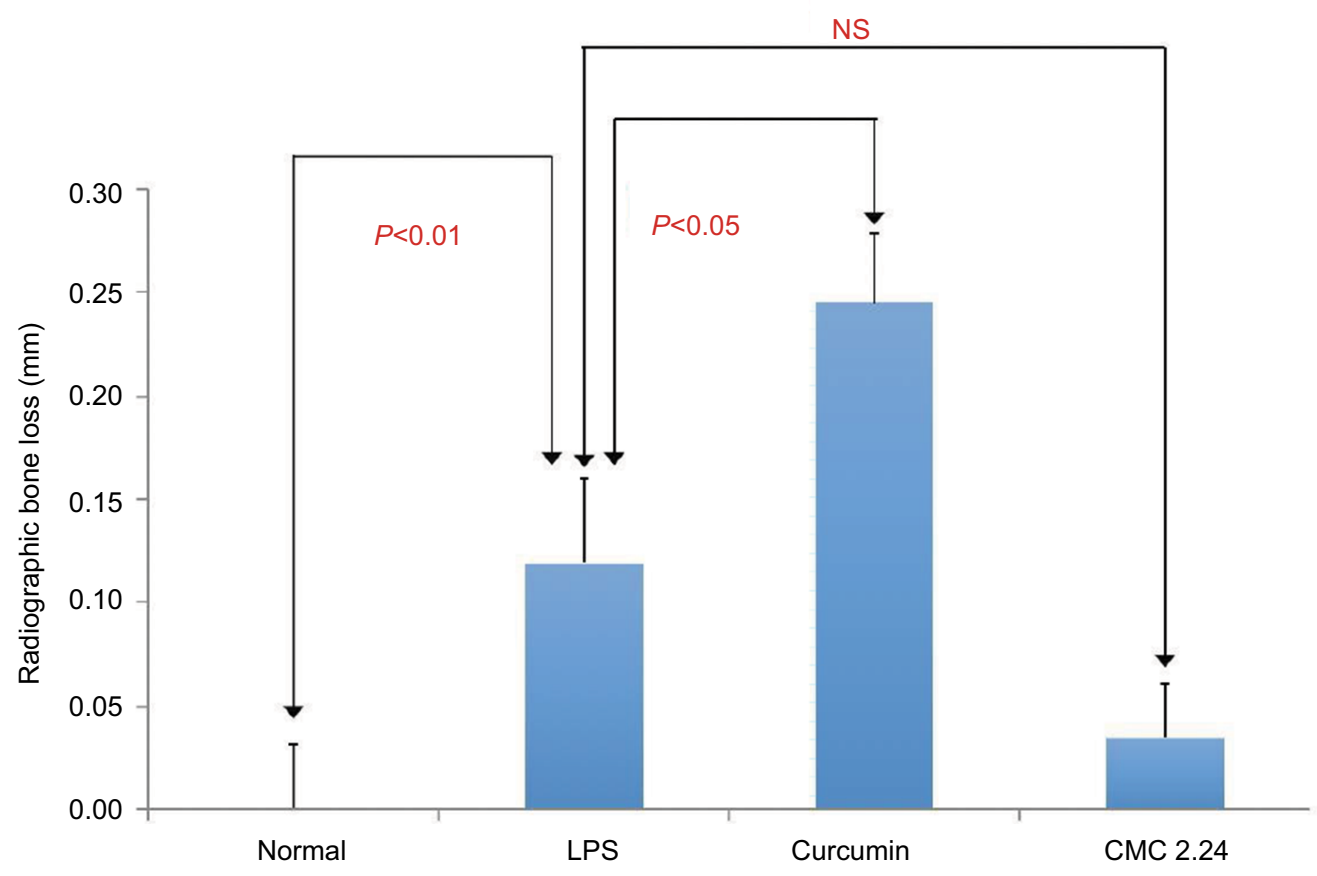

Figure 14 Radiographic analysis of maxillary bone loss at positions 7 and 8 between the first and second molars.

Notes: The maxillae were defleshed, and bone loss between first and second molars was assessed radiographically using EMAGO dental imaging software at positions 7 and 8. Alveolar bone loss is defined as the distance between the CEJ and the alveolar bone crest.

Abbreviations: CEJ, cemento-enamel junction; CMC, chemically modified curcumin; LPS, lipopolysaccharide; NS, not significant.

2.24 was found to show superior potency in vitro (ie, the lowest $\mathrm{IC}_{50}$ ) against various MMPs that are associated with tissue degradation. ${ }^{17}$ When CMC 2.24 was systemically administered to rats via oral gavage, a dosage of $30 \mathrm{mg} / \mathrm{kg}$ was found to reduce the secretion of pathological levels of MMP-9 with no evidence of toxicity. In these studies, and in previous experiments, treatment with CMC 2.24 did not show any evidence of toxicity based on cell cytotoxicity assay, and we observed no reduction in body weight gain with time, even in diabetic rats. ${ }^{24,33}$

Early on, a pilot study utilizing a minimal number of rats and a "split-mouth" experimental design found that CMC 2.24 was able to reduce both inflammatory mediator levels as well as clinical alveolar bone loss. ${ }^{24}$ However, this split-mouth design raised concerns that LPS injections in the gingiva can affect the overall systemic health of the rats, which may affect the PBS injection sites (controls) that were in the contralateral side of the same rat. In addition, in these studies, no comparisons between the efficacy of natural curcumin and CMC 2.24 on the treatment of periodontitis were made. The current study was designed to use individual rats rather than a split-mouth design in each group to eliminate the possible detrimental health effects of LPS injections in the control sites and the possible systemic cross-over benefits of orally administered CMC 2.24 or curcumin. In addition to comparing the therapeutic effects of CMC 2.24 to curcumin in the rat experimental periodontitis model, a cell culture assay assessing the drugs' effects on MMP-9, proinflammatory mediators, and cytokines was also performed.

In the current study, evidence of disease initiation and progression after 2 weeks of repeated LPS endotoxin injection was seen based on the dramatic increase of MMP-2 and MMP-9, IL-1 $\beta$, and most importantly, the significant clinical evidence of alveolar bone loss in the LPS-injected rats. However, reductions of both MMP-2 and MMP-9 to levels similar to or even below the normal group were observed in both the plasma (systemic circulation) and the gingiva of these groups when treated with CMC 2.24. It is notable that although the objective of this study was to assess the efficacy of CMC 2.24 in the treatment of local periodontal disease, the plasma levels of MMP-9 were also significantly lowered by CMC 2.24 but not by curcumin, indicating a more profound effect of CMC 2.24 on systemic complications of severe periodontal disease. This systemic benefit of CMC 2.24 may be due to the fact that (i) the experimental drug is systemically administered via oral gavage and (ii) that by reducing local periodontal inflammation, systemic inflammation is also decreased. This idea is supported by past research in treating diabetic rats with $\mathrm{CMC} 2.24$, which attenuated both local and systemic inflammation with a subsequent decrease 
of the diabetic complications. ${ }^{19}$ Of extreme interest, the profound suppression of excessive MMP-9 in the circulation of the rats treated with CMC 2.24 indicates a reduced risk for cardiovascular disease and other medical disorders. ${ }^{34}$ This could also reduce the risk for type II diabetes, since excess systemic MMPs can degrade the insulin receptor. ${ }^{35}$

The reduction of proinflammatory mediators and cytokines was observed in both the cell culture study as well as in vivo. In the cell culture study, TNF- $\alpha$, IL-1 $\beta$, and MMP-9 were reduced significantly with CMC 2.24 , while in the animal study, IL-1 $\beta$ was substantially reduced in gingival tissue when compared with that in the LPS/vehicle group. Similar to previous studies, both TNF- $\alpha$ and IL- 6 cytokines did not significantly change across the groups in either the plasma or the serum. ${ }^{24}$ However, TNF- $\alpha$ is much less potent than IL-1 $\beta$ in key aspects of periodontal disease pathogenesis with respect to their ability to stimulate bone resorption. ${ }^{36}$ Since IL- 6 acts as both a longer term proinflammatory and anti-inflammatory cytokine, the balance of IL-6 is essential in the progression and resolution of periodontal disease. ${ }^{37}$ With this in mind, it may be beneficial for the host if IL-6 levels were reduced to lower than that observed in the disease state but also not so much that IL-6 cannot act as a protective cytokine with its anti-inflammatory capabilities.

Aside from the analysis of cytokines and MMPs, the ultimate therapeutic outcome of alveolar bone loss, at the site of LPS-induced periodontitis, was assessed both morphometrically and radiographically. As previously mentioned, the location of interest is between the maxillary first and second molars - labeled sites 6, 7, 8, and 9 as described by Chang et al. ${ }^{30}$ In this analysis, the bone level of the normal group was set to zero in order to calculate the increased or decreased bone loss in the experimental groups. In the morphometric analysis, the CMC 2.24-treated group consistently exhibited decreased alveolar bone loss compared to the LPS/vehicle group. There was a pronounced and significant reduction of $86 \%$ in bone loss in the LPS/CMC 2.24 group compared to that in the LPS/vehicle group. These reductions were also seen in the radiographic analysis which was performed independently of the morphometric analysis. Although no statistically significant differences were seen in the bone density $(P>0.05)$, a beneficial trend was apparent in that there was a slight increase in bone density in the CMC 2.24-treated group compared to that in the LPS/vehicle group. In the past, our laboratory has examined this parameter in the diabetic rat model and found that those severely hyperglycemic animals exhibited decreased density in the skeletal long bones and alveolar bone, which was prevented by systemic administration of CMC 2.24. The density changes in the current study may not be apparent due to its short duration or due to the nature of this animal model. It is also known that the alveolar bone more readily undergoes collagen degradation and turnover, which may also play a role in the increased threshold for detection of bone density changes. ${ }^{38}$

In comparing CMC 2.24 to curcumin, the former was more efficacious in reducing (a) MMP-9 levels in both the cell culture and animal studies and (b) alveolar bone loss in rats with periodontitis. It is of interest to note that in this study, excessive alveolar bone loss was seen in the curcumintreated group compared to that in the control group. These results support the observations of Guimarães et al who found that systemic administration of natural curcumin is capable of decreasing proinflammatory markers but cannot inhibit bone loss in periodontitis as measured by micro-CT imaging. ${ }^{23}$ However, there are other studies demonstrating that curcumin can inhibit alveolar bone loss in experimental periodontitis. Correa et al showed that daily oral gavage with curcumin for 30 days was able to significantly, but minimally decrease alveolar bone loss by $0.08 \mathrm{~mm}$ or $5.7 \%(1.33 \mathrm{~mm}$ in curcumin group vs $1.41 \mathrm{~mm}$ in the control group). ${ }^{39} \mathrm{~A}$ more recent study by Bakir et al showed that curcumin is a promising host modulatory agent and was able to reduce alveolar bone loss by more than $27 \%{ }^{40}$ This may be due to the intrinsic dose-dependent cytotoxicity of curcumin, which can induce the generation of reactive oxygen species, causing cellular damage and cellular apoptosis, ${ }^{41-43}$ or maybe a longer course of curcumin administration will be needed to exhibit its reduction on bone loss. Additionally, different experimental models of periodontitis may have led to the varying results as evidenced by the previously mentioned studies..$^{23,39,40}$

In conclusion, this study demonstrates that oral administration of CMC 2.24 leads to inhibition of proinflammatory cytokines and MMPs, and reduces pathologic alveolar bone loss in rat experimental periodontitis. Within the limitations of this study, it was found that treatment with curcumin can decrease proinflammatory mediators and cytokines but cannot reduce (possibly even accelerate, although this unexpected observation requires substantiation) alveolar bone loss. In this study, systemic administration of $30 \mathrm{mg} /$ $\mathrm{kg}$ curcumin slightly increased alveolar bone loss with borderline significance.

The results indicate that CMC 2.24 is effective in the prevention of rat experimental periodontitis in the "prophylactic model." Future studies will examine whether alreadyestablished periodontitis will also respond favorably to CMC 2.24 treatment, similar to treatment of patients with diagnosed chronic periodontitis in clinical practice, or what would be considered the "therapeutic model." 


\section{Acknowledgments}

This research is supported by US National Institutes of Health/ National Institute of Dental and Craniofacial Research grant number 1R41DE024946-01 and Traverse Biosciences, Inc.

\section{Disclosure}

F Johnson and LM Golub have several patents on CMC 2.24. For LM Golub, these are fully assigned to SBU. For F Johnson, these are assigned to ChemMaster, Intl. J Scaduto is employed by Traverse Biosciences Inc., Stony Brook, NY, USA. The authors report no other conflicts of interest in this work.

\section{References}

1. Ximénez-Fyvie LA, Haffajee AD, Socransky SS. Comparison of the microbiota of supra- and subgingival plaque in health and periodontitis. J Clin Periodontol. 2000;27(9):648-657.

2. Ryan ME. Host modulation: conceptualization to clinical trials and integration into clinical practice. J Calif Dent Assoc. 2002;30(4):285-288, 290-295.

3. Kornman KS. Host modulation as a therapeutic strategy in the treatment of periodontal disease. Clin Infect Dis. 1999;28(3):520-526.

4. Pihlstrom BL, Mchugh RB, Oliphant TH, Ortiz-Campos C. Comparison of surgical and nonsurgical treatment of periodontal disease. A review of current studies and additional results after 61/2 years. J Clin Periodontol. 1983;10(5):524-541.

5. Ryan ME. Nonsurgical approaches for the treatment of periodontal diseases. Dent Clin North Am. 2005;49(3):611-636.

6. Isola G, Matarese G, Williams RC, et al. The effects of a desiccant agent in the treatment of chronic periodontitis: a randomized, controlled clinical trial. Clin Oral Investig. 2018;22(2):791-800.

7. Pirracchio L, Joos A, Luder N, Sculean A, Eick S. Activity of taurolidine gels on ex vivo periodontal biofilm. Clin Oral Investig. 2018;22(5):2031-2037.

8. Beacher N, Sweeney MP, Bagg J. Dentists, antibiotics and Clostridium difficile-associated disease. Br Dent J. 2015;219(6):275-279.

9. Walker CB. The acquisition of antibiotic resistance in the periodontal microflora. Periodontol 2000. 1996;10(10):79-88.

10. Gu Y, Walker C, Ryan ME, Payne JB, Golub LM. Non-antibacterial tetracycline formulations: clinical applications in dentistry and medicine. J Oral Microbiol. 2012;4(1):19227.

11. Mckeage K, Deeks ED. Doxycycline $40 \mathrm{mg}$ capsules $(30 \mathrm{mg}$ immediate-release $/ 10 \mathrm{mg}$ delayed-release beads): anti-inflammatory dose in rosacea. Am J Clin Dermatol. 2010;11(3):217-222.

12. Golub LM, Lee HM, Greenwald RA, et al. A matrix metalloproteinase inhibitor reduces bone-type collagen degradation fragments and specific collagenases in gingival crevicular fluid during adult periodontitis. Inflamm Res. 1997;46(8):310-319.

13. Golub LM, Mcnamara TF, Ryan ME, et al. Adjunctive treatment with subantimicrobial doses of doxycycline: effects on gingival fluid collagenase activity and attachment loss in adult periodontitis. $J$ Clin Periodontol. 2001;28(2):146-156.

14. Aravind SR, Krishnan LK. Curcumin-albumin conjugates as an effective anti-cancer agent with immunomodulatory properties. Int Immunopharmacol. 2016;34:78-85.

15. Gupta SK, Kumar B, Nag TC, et al. Curcumin prevents experimental diabetic retinopathy in rats through its hypoglycemic, antioxidant, and antiinflammatory mechanisms. JOcul Pharmacol Ther. 2011;27(2):123-130.

16. Soetikno V, Sari FR, Sukumaran V, et al. Curcumin prevents diabetic cardiomyopathy in streptozotocin-induced diabetic rats: possible involvement of PKC-MAPK signaling pathway. Eur J Pharm Sci. 2012;47(3):604-614.
17. Zhang Y, Gu Y, Lee HM, et al. Design, synthesis and biological activity of new polyenolic inhibitors of matrix metalloproteinases: a focus on chemically-modified curcumins. Curr Med Chem. 2012;19(25): 4348-4358.

18. Botchkina GI, Zuniga ES, Rowehl RH, et al. Prostate cancer stem cell-targeted efficacy of a new-generation taxoid, SBT-1214 and novel polyenolic zinc-binding curcuminoid, CMC2.24. PLoS One. 2013;8(9):e69884.

19. Elburki MS, Moore DD, Terezakis NG, et al. A novel chemically modified curcumin reduces inflammation-mediated connective tissue breakdown in a rat model of diabetes: periodontal and systemic effects. J Periodontal Res. 2017;52(2):186-200.

20. Xu Y, Ge L, Abdel-Razek O, et al. Differential susceptibility of human Sp-B genetic variants on lung injury caused by bacterial pneumonia and the effect of a chemically modified curcumin. Shock. 2016;45(4):375-384.

21. Zhang Y, Mcclain SA, Lee HM, et al. A Novel Chemically modified curcumin "Normalizes" wound-healing in rats with experimentally induced type I diabetes: initial studies. J Diabetes Res. 2016;2016:11.

22. Sergaeva A, Wang R, Trochesset D, Colosi D, London S, London L. Inhibition of initiation and progression of periodontal disease by curcumin. AADR Annual Meeting 2012. Tampa, Florida, USA: American Association for Dental Research; 2012.

23. Guimarães MR, Coimbra LS, de Aquino SG, Spolidorio LC, Kirkwood KL, Rossa C. Potent anti-inflammatory effects of systemically administered curcumin modulate periodontal disease in vivo. J Periodontal Res. 2011;46(2):269-279.

24. Elburki MS, Rossa C, Guimaraes MR, et al. A novel chemically modified curcumin reduces severity of experimental periodontal disease in rats: initial observations. Mediators Inflamm. 2014;2014:959471.

25. Vardar S, Buduneli E, Türkoğlu O, et al. Therapeutic versus prophylactic plus therapeutic administration of omega- 3 fatty acid on endotoxininduced periodontitis in rats. J Periodontol. 2004;75(12):1640-1646.

26. Golub LM, Evans RT, Mcnamara TF, Lee HM, Ramamurthy NS. A nonantimicrobial tetracycline inhibits gingival matrix metalloproteinases and bone loss in Porphyromonas gingivalis-induced periodontitis in rats. Ann N Y Acad Sci. 1994;732:96-111.

27. Ramamurthy NS, Golub LM. Diabetes increases collagenase activity in extracts of rat gingiva and skin. J Periodontal Res. 1983;18(1):23-30.

28. Hu X, Beeton C. Detection of functional matrix metalloproteinases by zymography. Journal of Visualized Experiments. 2010;(45):2445.

29. Zhang Y, Golub LM, Johnson F, Wishnia A. pKa, zinc- and serum albumin-binding of curcumin and two novel biologically-active chemically-modified curcumins. Curr Med Chem. 2012;19(25):4367-4375.

30. Chang KM, Ramamurthy NS, Mcnamara TF, et al. Tetracyclines inhibit Porphyromonas gingivalis-induced alveolar bone loss in rats by a nonantimicrobial mechanism. J Periodontal Res. 1994;29(4):242-249.

31. Bulkley D, Johnson F, Steitz TA. The antibiotic thermorubin inhibits protein synthesis by binding to inter-subunit bridge B2a of the ribosome. J Mol Biol. 2012;416(4):571-578.

32. Johnson F, Chandra B, Iden CR, et al. Thermorubin 1. Structure studies. JAm Chem Soc. 1980;102(17):5580-5585.

33. Elburki MS, Moore DD, Terezakis NG, et al. A novel chemically modified curcumin reduces inflammation-mediated connective tissue breakdown in a rat model of diabetes: periodontal and systemic effects. J Periodontal Res. 2017;52(2):186-200.

34. Blankenberg S, Rupprecht HJ, Poirier O, et al. Plasma concentrations and genetic variation of matrix metalloproteinase 9 and prognosis of patients with cardiovascular disease. Circulation. 2003;107(12):1579-1585.

35. Fischoeder A, Meyborg H, Stibenz D, Fleck E, Graf K, Stawowy P. Insulin augments matrix metalloproteinase-9 expression in monocytes. Cardiovasc Res. 2007;73(4):841-848.

36. Stashenko P, Dewhirst FE, Peros WJ, Kent RL, Ago JM. Synergistic interactions between interleukin 1, tumor necrosis factor, and lymphotoxin in bone resorption. J Immunol. 1987;138(5):1464-1468.

37. Irwin CR, Myrillas TT. The role of IL- 6 in the pathogenesis of periodontal disease. Oral Dis. 1998;4(1):43-47. 
38. Matsuura T, Tokutomi K, Sasaki M, Katafuchi M, Mizumachi E, Sato H. Distinct characteristics of mandibular bone collagen relative to long bone collagen: relevance to clinical dentistry. Biomed Res Int. 2014;2014:769414.

39. Correa MG, Pires PR, Ribeiro FV, et al. Systemic treatment with resveratrol and/or curcumin reduces the progression of experimental periodontitis in rats. J Periodontal Res. 2017;52(2):201-209.

40. Bakır B, Yetkin Ay Z, Büyükbayram Hİ, et al. Effect of curcumin on systemic $\mathrm{T}$ helper 17 cell response; gingival expressions of interleukin-17 and retinoic acid receptor-related orphan receptor $\gamma \mathrm{t}$; and alveolar bone loss in experimental periodontitis. J Periodontol. 2016;87(11):e183-e191.
41. Atsumi T, Tonosaki K, Fujisawa S. Comparative cytotoxicity and ROS generation by curcumin and tetrahydrocurcumin following visible-light irradiation or treatment with horseradish peroxidase. Anticancer Res. 2007;27(1A):363-371.

42. Kunwar A, Barik A, Mishra B, Rathinasamy K, Pandey R, Priyadarsini KI. Quantitative cellular uptake, localization and cytotoxicity of curcumin in normal and tumor cells. Biochim Biophys Acta. 2008;1780(4):673-679.

43. Woo JH, Kim YH, Choi YJ, et al. Molecular mechanisms of curcumininduced cytotoxicity: induction of apoptosis through generation of reactive oxygen species, down-regulation of Bcl-XL and IAP, the release of cytochrome $\mathrm{c}$ and inhibition of Akt. Carcinogenesis. 2003;24(7):1199-1208.

\section{Publish your work in this journal}

The Journal of Experimental Pharmacology is an international, peerreviewed, open access journal publishing original research, reports, reviews and commentaries on all areas of laboratory and experimental pharmacology. The manuscript management system is completely online and includes a very quick and fair peer-review system.
Visit http://www.dovepress.com/testimonials.php to read real quotes from published authors. 